\title{
Analysis on the Costume Art of Xinping Huayao Dai*
}

\author{
Danting Sun \\ School of Art and Design \\ Wuhan University of Technology \\ Wuhan, China 430070 \\ School of Fine Arts \\ Yuxi Normal University \\ Yuxi, China 653100
}

\author{
Ping Wen \\ School of Mathematics and Information Technology \\ Yuxi Normal University \\ Yuxi, China 653100
}

\author{
Xiaobo Ren \\ School of Art \\ Wenshan University \\ Wenshan, China 663099
}

\begin{abstract}
The Dai nationality is one of the ethnic minorities in China with a large population and deep culture. Huayao Dai which lived in Xinping County, Honghe basin of Yunnan province, is distinguished from other Dai people by its distinctive clothing form. Based on a large number of field surveys, this paper analyzes the structural characteristics of the clothing of Daiya, Daisa and Daika three Huayao Dai branches.
\end{abstract}

Keywords-Huayao Dai; branch; garment structure

\section{INTRODUCTION}

During the long history development process, various ethnic groups in Yunnan have developed their own unique dress styles. Dai nationality is one of the many ethnic minorities in China with a long history and rich culture. At present, there are Dai ethnic groups lived in the Lancang, Nujiang, Honghe and Jinsha river basins in Yunnan. As a special group among Dai nationality, the Huayao Dai has multiplied and thrived one generation after another in the Honghe Valley which in the southwest of China and under the Ailao Mountain.

\section{OVERVIEW OF HUAYAO DAI}

In the Xinping Yi-Dai Autonomous County, about 50,000 Dai people live together in the area of 70 kilometers long, 7 kilometers wide, about 500 square kilometers from Gasa River to Mosha River, it has the most Dai people lived together among all the counties along the Honghe River. The Dai people are divided into different branches based on the names of living places, the characteristics of historical migration, the characteristics of clothing, and the customs. Most of the Dai costumes in Xinping County share a common feature, there is

*Fund Project: Humanities and Social Sciences Research Project of the Ministry of Education "Research on the productive protection of the Costume Culture and Traditional craftsmanship of Xinping Huayao Dai" (Project Number: 17YJC760071) a long colored cotton belt wrapped around their waists, so they are known as "Huayao Dai". There are also three selfproclaimed names: "Daiya", "Daika", and "Daisa". The ancient Dai language called Mosha as "Mengya", which means the living place left by the migrants from the migration journey. "Daiya" is the residence of Dai people who live in Mengya, and it is the place left by Dai people in the migration process. Gasa and water pond referred as "Mengsa" in ancient Dai language, "Sa" means beach, and "Daisa" means Dai people who lived by the river bank. Daika, "Ka" referred as other nationalities besides Dai in ancient Dai language, "Daika" means the Dai nationality that evolved from other ethnic groups. It is mainly distributed in the fish ponds, dragon river, Xini of Xinping and Manbeng, Ede, Modao of Yaojie and other places.

\section{GARMENT STRUCTURE AND ARTISTIC FEATURES}

Compared with other national costumes, Huayao Dai costumes have their commonalities as well as their own characteristics, especially the color matching of costumes and styles.

\section{A. Daiya Branch}

The form of Daiya Women's inner wear is a stand collar slanted coat. Whether it is the daily dress of the middle-aged and the elderly or the fancy dress of young women, the under clothes are all sleeveless, straight lapel, and right front. The under clothes of the three branches of Huayao Dai are all right front, and according to history records, traditional Han clothing was recorded as right front while ethnic minorities were mostly left front, however, the minorities living in Yunnan are basically right front, Huayao Dai is no exception. From this we can see that since ancient times, the influence of inter-ethnic exchanges has been truly reflected in the clothing style. The neckline of the Daiya clothing has already noticed 
the columnar structure of human neck, so the arc of the collar has been cut into an arc shape, but the waist is still straight. The armholes are cut and the modern youth women's dresses have been cut into arcs in accordance with the cylindrical shape of the arms, while the middle-aged and elder clothes still maintain the straight line cuts on the sleeves. On the shoulder line cutting, the dress of the young woman has already noticed the inclination of shoulder, so they use the form of oblique lines, while the middle-aged and the elder clothes are still parallel straight shoulder lines. Daiya Women's outerwear is a straight-collar front opening style. Whether it is the outerwear of middle-aged and elder people, or the dress of young women, both left the column sized width which the collar required on the front and rear shoulder lines, and then the forged fabrics, brocades or embroidered pieces, silver blister or silver sesame bells are used to quilted straight collar. After the outerwear is worn, the shoulder line of the clothes is naturally pushed back by the pressure from the three-dimensional neck, which causes the front opening be lifted up. Because of the thickness of the collar, the weight of the silver blister and the silver sesame bell make the front opening up, and the weight of the clothes are all pressed on the chest, and the back of the clothes is falling due to overall backward movement.[1]

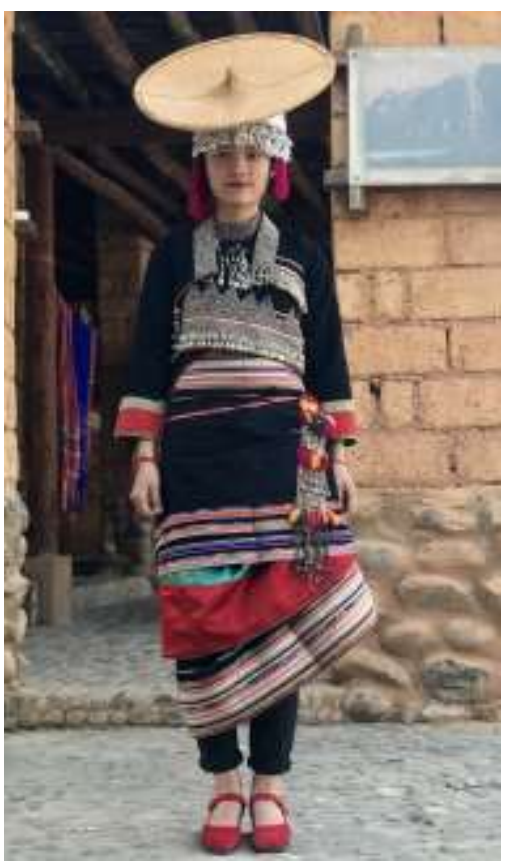

Fig. 1. Front side of Daiya Costume.

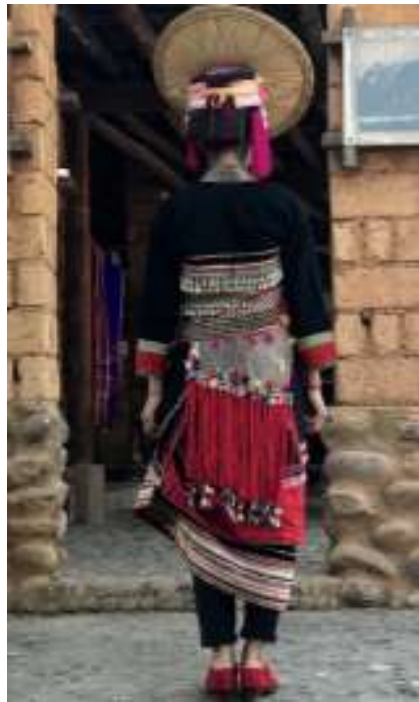

Fig. 2. Back side of Daiya Costume.

The outerwear of Daiya have narrow sleeves, no button, straight collar, and front opening, the neckline is shaped as linear, after the collar is unfolded, the structure is rectangular. After put on the coat, it shows a unique triangular collar shape, this combination collar type is different with the modern collar's three-dimensional shape, it shows a flat regularity and is very conducive to repeated embroidery on the collar. The outerwear is slightly shorter than the under clothes and can only cover the chest, so it can just reveal the under clothes which is covered with silver blisters and silver sesame bells. The sleeves are slender to the wrists, and the sleeves are folded toward the cuffs. There are broken seams in the sleeves and wide coloured silk splicing at the cuffs. Satin strips with different colors and different width are also spliced before and after the hem. In the front, three silver blisters arranged in parallel on both the left and right sides of the colored satin strips, and then a row of silver sesame bells are affixed under the silver blisters. The back and the front piece are embedded at the same height with a continuous arrangement of triangular silver blister, and below the silver blister is also accompanied by a row of silver sesame bells.

The tube skirt of Daiya is stitched by a piece of homemade black homespun. The tube skirt is divided into two parts, the upper part is a self-woven black homespun, lower part is composed of a wide colored silk and embroidery and three small parts. The upper part is green, the middle is red, and the lower part is made up of laces inlaid with multiple colors, the pattern can be freely chosen without restriction. According to the interpretation of local residents in the field survey, the green part symbolizes the leaves or the forest, the red part symbolizes the fruit, and the lace inlaid with multiple colors symbolizes the rainbow. After the tube skirt has been folded and stitched, the upper half of the side seam is left with a slit and decorated with a homemade colored strip to fix the skirt. Tube skirt should be worn from top to bottom, if worn from bottom up, it is considered unsightly behavior. When wearing the skirt, lift the left side of the skirt and stuffed to the waist and fasten it with homemade colored strip. The waistband of Daiyae is sewn from a rectangular navy blue fabric piece, 
colored stain strips are trimmed around the waistband, colored embroidery are on the hem, and both sides of the waistband also have colored embroidery, even at the back of the waistband there are also colored decorative ribbon patterns, basically do not see rough edges, so it has fine workmanship. In the past, there were also Huayao belt in the dresses of Daiya. Now, in order to make them easy to wear, it replaced with a wide ribbon of silk satin cloth which attached to the area around the waistband. Daiya's leggings are a piece of navy blue cloth about half a meter long and about one meter long. There are red strings at both ends, and some of them have embroidery.

Headwear: The long hair is twisted into round shape, coiled together at the top of head and presented as flat-topped cone. A blue cloth is folded neatly around the top of the head from the bun's feet, the top of the round, shiny hair bun is exposed outside the hat (it is called Nei bao tou), and then use a threefinger wide blue cloth folded into a small and unique band and cover both ends of the ear from the top of the head, it was called "ear band". Every festival it will be festooned with a bunch of colorful enamels, and then covered with a piece of lace turban with green, red, purple, yellow, and green stripe that is more than a foot wide from the forehead toward back (it is called Wai bao tou), wearing silver earrings it will become a headwear. When you leave home, you'll need to wear a bamboo hat shaped like a blossoming termitomyces albuminosus.

\section{B. Daisa Branch}

Daisa women's inner wear is special, belonging to the collarless slanted type. The back collar line has obvious undercuts. Just as Daiya's inner wear, Daisa also noticed the three-dimensional structure of human neck, making it more comfortable to wear. At the same time, it makes narrow brocade stitching along the arc of the collar, so that the collar structure is even more obvious. One of the reasons for this design is that the neck is often subject to friction, so it needs to be reinforced; The other theory is it makes the color more beautiful and bright. The placket is right front and slanted type, decorated with embroidered cross-stitched linings, silver blisters are used to decorate the plackets, and in the meantime contrast the placket in order to make it beautiful. [1]

Middle-aged and elderly women's inner wear is similar to young people, except that there is a difference in fabric selection. Middle-aged and elderly women mainly use black cotton, while young women are mainly use silk fabric in various colors. The structure of the Daisa middle-aged women and young women's outerwear are the same, except that there is a difference in the use of fabrics. The fabric of outer wear is the same as inner wear, young women often use the silk fabrics woven by modern machines, and middle-aged and elderly people often use modern machine-made cotton cloth or homemade homespun. The front and back of the jacket are a whole fabric, and stitching is carried out on the back piece, the front piece is sewn along the placket with a woven tape fabric to form a straight collar that connect collar to the placket. After the outerwear is worn, the shoulder line of the clothes is naturally pushed back by the pressure from the threedimensional neck, which causes the front opening be lifted up and form a natural overlapping crossover on the chest, and the back fall downwards and outwards, the fabric around the neckline form a clear fold. [2]

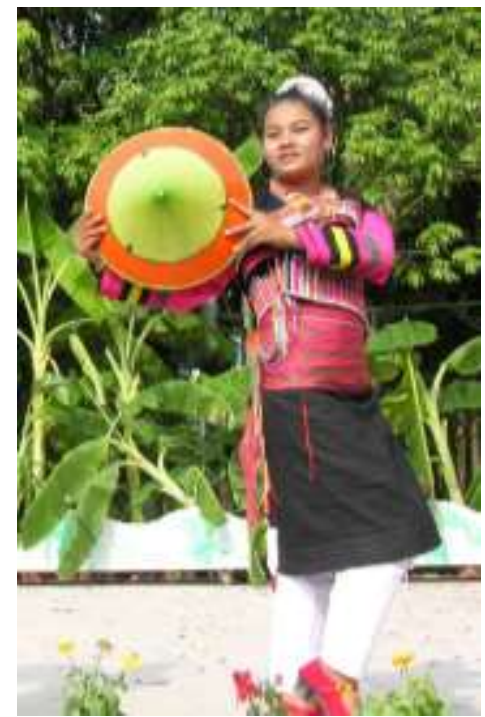

Fig. 3. Front side of Daisa Costume.

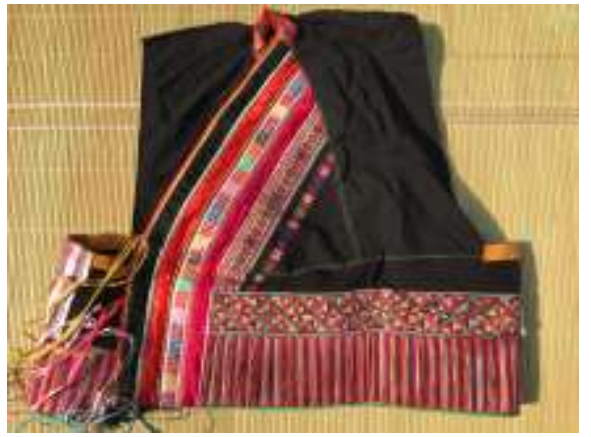

Fig. 4. Under clothes of Daisa.

The lower skirt of Daisa costumes is slightly different from Daiya and Daika. It is composed of a tube skirt and leggings. The Daisa woman's costume originally has a waistband, now it is basically gone, only the middle-aged and elderly people will use blue cotton apron while working. The Daisa's tube skirt is basically black silk satin decorated with patterns, after the skirt is sewn, it is a piece of square cloth and there is no decoration at the waist, the geometric cross-stitch pattern is stitched at the hem, rows of sequins are arranged in the middle of the pattern as decoration. When wearing it, put it on the waist from top to bottom, it is sewn on the left side, and it is fixed on the waist with a Huayao band to form a left high and right low shaped hem. Daisa's leggings are also different from Daiya and Daika. Daisa's leggings are cylindrical, up wide and lower narrow, they can be directly put on the calf when they are used, then use knitted colored string to fix them at the knee. Young women's leggings are made of colored silk fabric, middle-aged and elderly people's leggings are made of white polyester fabrics.

Headwear: All the hair is also twisted into round shape, coiled together at the back of head and presented as oblique flat-topped cone, at the top of the cone covered with color- 
matched towel which fold down with edge, use blue head scarf with red, white, yellow, and green colors to wrap the hair behind the head, only reveal the forehead hair. At the foot of the hairpin, use a three-finger-width blue cloth as lace, and a large sliver blister in the middle is attached to the back of the head, and wear a bamboo hat shaped like termitomyces albuminosus follicle and with a round top rounded to form a round beak. This is the headwear of Daisa

\section{Daika Branch}

Daika women's inner wear is collarless slanted type, but it is different from Daisa's collarless slanted type. Daika's inner wear is a right front straight style, and the neckline does not have the tendency for down digging, nor does it require the width of the neck on the front and rear shoulder lines as in Daiya's jacket. The right part of the piece is just a piece of rectangular brocade fabric on the left front piece based on the front piece. The inner piece is still straight. Therefore, the pleats are formed around the collar due to the compression of the neck. Daika can also be influenced by Daiya, some inner wear shapes are also collarless slanted type like Daiya, but only in the stitching of the right piece, Daika is wider than the Daiya's splicing fabric. The shape of Daika Women's outerwear is straight collar front opening just like Daiya, but the hem of the cloth and the pocket are as wide as the inner wear. In terms of fabric collocation, Daika is also different from Daiya, Daika mostly uses self-weaving homespun fabrics (or machine-woven black cotton fabrics) in combination with machine-made cotton (or polyester-cotton) fabrics, while Daiya mostly uses black cotton fabrics in combination with high-gloss silk forgings, so from the overall visual aspect, Daika is slightly more subtle than Daiya. Daika's tops are much richer in the use of tapestry, the back piece is adorned with a large area of colored tapestry, so it is very eye-catching, and Daiya mostly decorated with silver blisters and silver sesame bells. [3]

Daika's outer wear is similar to Daiya, it is straight collar, front opening, and short-sleeved jacket. The front panel is lined with triangular and diamond-shaped silver blister; the hem of front panel is stitched with white, red, blue, pink and green colored cotton strips. At the arm sleeve area, where the seams of the sleeves are stitched, a decorative flower pattern is used to decorate the interior. The inside of the cuffs is decorated with white, blue and pink cotton cloths. When worn, the cuffs can be raised to reveal colored stripes, and it is also convenient for the field work. The back of the jacket, from the collar down to the hem, strips embroidered with various shapes of colorful patterns are spliced on the back and the lower half are decorated with colored brocades.

The lower wear of Daika's costume is also composed of tube skirt, waistband and leggings like Daiya. Daika's tube skirt is folded in a rectangular shape. From the overall point of view, it is divided into two parts, the upper part is a self-woven black homespun cloth, and the lower part is a combination of colorful cross-stitch and brocades. When wearing the tube skirt, from left to right, there are some wavy ruffles. Daika's waistband is a navy blue cotton cloth in appearance, a piece of colorful tapestry is put on the inside of the top of waistband. When it is worn, the tapestry is folded down to expose the bright tapestry on the outside; both sides of the waistband are embroidered with cross-stitch patterns, and the hem is also decorated with embroidery patterns. The leggings of Daika are similar to those of Daiya, on one side of the cyan fabric, it embroidered with a cross-stitch pattern about $3 \mathrm{~cm}$ wide, and they will be exposed on the outside as decoration after wrap.
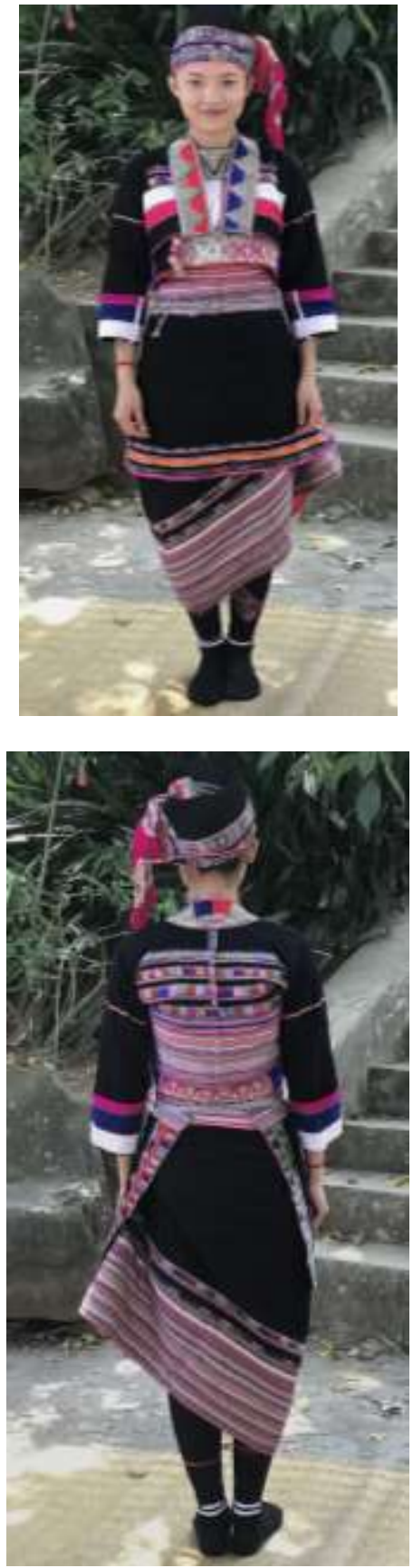

Fig. 5. Front and Back side of Daika Costume.

Headwear: Daika's headwear is to twist long hair into updo on the top of the head, use a piece of green cloth about seven inches wide and five feet long, with cross-stitch patterns on the edge and on the corner of the cloth there are silk tassels. When wrapping the head, wrap the towel from left to right one circle, covering the updo, dragging the left corner and fold in the 
seam of the ear dome, and the cross-stitch pattern and tassels of the towel will hold down from the top of the head to the left ear. When left the house, they will wear a spire and a flatbrimmed hat. When they go the street to visit family, they will wear a tung oil bamboo hat and silver necklaces. The special hat was also fitted with a round mirror.

\section{CONCLUSION}

The type, style and color of costume are intrinsically linked to the historical and cultural system to which each nation belongs, the natural environment of the society, the economic life style, the national psychology, and the aesthetic interest. It is a comprehensive product of many cultural factors.

The beauty of Huayao Dai costume not only comes from the color and shape, but also comes from the charm formed by the combination of color and shape. The kind of personal characteristics that transcend the external sensibility image expressed by Hanyao Dai's costume, the unceasing artistic appeal and the vitality of beauty are incomparable to other ethnic groups.

It should be said that costume is not a simple body protection issue, but a reflection of society, a nation, and a region's social consciousness and cultural mentality. Costume soaked in the wisdom and sweat of a nation, documenting a nation's tireless spirit of exploration and the ability to build life. [4]

\section{REFERENCES}

[1] Yang Xiaomi. Research on the costume structure of Yunnan Xinping Huayao Dai [D]. Kunming: Master thesis of Kunming University of Science and Technology, 2014:26-27. 杨晓密. 云南新平花腰傣服饰结 构研究[D].昆明: 昆明理工大学硕士论文, 2014: 26-27

[2] Ibid.,28

[3] Ibid.,29

[4] Li Yongxiang, Chief Editor. Huayao Dai Costume Art and Intangible Cultural Heritage Protection: The Proceedings of China Huayao Dai Costume Culture Academic Symposium [C]. Kunming: Yunnan Ethnic Publishing Company, 2008:52. 李永祥主编. 花腰傣服饰艺术与非物质 文化遗产保护: 中国花腰傣服饰文化学术研讨会论文集 [C]. 昆明: 云南民族出版社，2008: 52 